\title{
Letter to the editor Re: Faienza MF, Brunetti G, Grugni G, et al. The genetic background and vitamin $D$ supplementation can affect irisin levels in Prader-Willi syndrome. J Endocrinol Invest 2021 Mar 3. https://doi.org/10.1007/s40618-021-01533-4
}

\section{T. Kawada ${ }^{1}$ iD}

Received: 9 July 2021 / Accepted: 12 July 2021 / Published online: 19 July 2021

(c) Italian Society of Endocrinology (SIE) 2021

Dear editor,

Faienza et al. measured serum irisin concentration in children and adult patients with Prader-Willi syndrome (PWS) [1]. By multiple regression analysis, serum irisin levels in pediatric and adult PWS could be predicted by the genetic background and 25(OH)-vitamin D levels. In addition, intelligent indicator was selected as a significant predictor of serum irisin in adult patients with PWS. I present information on the association between serum irisin, bone health and cognition.

By a randomized controlled trial with combination of exercise and vitamin D supplementation, inverse relationship between pro-inflammatory marker and serum irisin concentration became stronger [2]. According to a review by Zhong et al., regulation of bone cell metabolism by irisin might be accelerated by exercise and vitamin D [3].

Conti et al. measured serum irisin and brain-derived neurotrophic factor (BDNF) in patients with Alzheimer's disease (AD) [4]. Mean serum irisin concentration was significantly elevated in patients with agitation/aggression (AA), and it correlated with the duration of AA. In contrast, there was no significant change in serum BDNF. They considered that serum irisin concentration might reflect the clinical status of $\mathrm{AD}$ in relation to aberrant motor behavior.

Among several biological action of circulating irisin, I suppose that changes in bone metabolism and cognition may be related in older subjects, and comprehensive studies are

This reply refers to the original article available at https://doi.org/ 10.1007/s40618-021-01533-4.

\section{T. Kawada}

kawada@nms.ac.jp

1 Department of Hygiene and Public Health, Nippon Medical School, 1-1-5 Sendagi, Bunkyo-Ku, Tokyo 113-8602, Japan needed to verify the inter-relationship among bone metabolism, cognition and serum irisin concentration.

Funding Nothing to declare.

\section{Declarations}

Conflict of interest The author declares that he has no known competing financial interests or personal relationships that could have appeared to influence the work reported in this paper.

Research involving human participants and/or animals This article does not contain any studies with human participants or animals performed by any of the authors..

Informed consent For this type of study, formal consent is not required.

\section{References}

1. Faienza MF, Brunetti G, Grugni G et al (2021) The genetic background and vitamin D supplementation can affect irisin levels in Prader-Willi syndrome. J Endocrinol Invest. https://doi.org/10. 1007/s40618-021-01533-4(Epub2021Mar3)

2. Gmiat A, Mieszkowski J, Prusik K et al (2017) Changes in proinflammatory markers and leucine concentrations in response to Nordic Walking training combined with vitamin D supplementation in elderly women. Biogerontology 18(4):535-548

3. Zhong X, Sun X, Shan M et al (2021) The production, detection, and origin of irisin and its effect on bone cells. Int J Biol Macromol 178:316-324

4. Conti E, Grana D, Stefanoni G et al (2019) Irisin and BDNF serum levels and behavioral disturbances in Alzheimer's disease. Neurol Sci 40(6):1145-1150

Publisher's Note Springer Nature remains neutral with regard to jurisdictional claims in published maps and institutional affiliations. 